Trauma Berufskrankh $2006 \cdot 8$ [Suppl 1]: S44 DOI 10.1007/s10039-005-1089-4

Online publiziert: 16. Februar 2006

๑) Springer Medizin Verlag 2006

F. Hahn

Ostalb-Klinikum, Klinikum der Zentralversorgung, Aalen

\title{
Schmerztherapie nach Trauma
}

\section{Diskussion des Vortrags von Prof. Dr. Klose, Ludwigshafen, mit dem Auditorium}

Patient erwarten - so weit die haftungsrechtliche Abgrenzung. Strafrechtsrelevant ist die Frage, ob eine unzureichende Schmerzbehandlung als unterlassene Hilfeleistung geahndet werden könnte. Darüber sind verschiedene Gremien durchaus unterschiedlicher Meinung. Betont werden muss aber erneut, dass es kein Recht auf „das schmerzfreie Krankenhaus“ gibt. Tägliches Bemühen um Schmerzreduktion muss aber für den Patienten deutlich erkennbar sein, muss auch eindeutig dokumentiert werden.

Die Regionalanalgesie, z. B. der Femoralisblock, als gute Maßnahme zur Reduktion von oralen oder parenteralen Schmerzmitteln erfordert einigen Aufwand. Fraglich ist, ob diese Methode nur stationären Patienten vorbehalten sein soll. In den USA werden bereits seit einigen Jahren Schmerzkatheter ambulant angewendet, diese Methode ist also ambulant durchführbar.

Die Frage, ob die Applikation von Schmerzkathetern nur den Anästhesisten vorbehalten ist, kann eindeutig verneint werden. Meistens legen die Operateure solche Schmerzkatheter in Kooperation und Absprache mit dem Anästhesisten in den Wundbereich.

Ob physiotherapeutische Dehnübungen an oder über der Schmerzgrenze in der postoperativen Behandlung als Impuls für das Schmerzgedächtnis bedenklich sind, konnte weder vom Panel noch vom Auditorium sachkundig geklärt werden.

\author{
Korrespondierender Autor \\ Prof. Dr. F. Hahn \\ Ostalb-Klinikum, Klinikum der \\ Zentralversorgung \\ Im Kälblesrain 1, 73428 Aalen \\ prof.hahn@ostalb-klinikum.de
}

